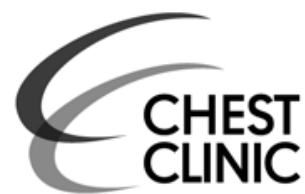

- Additional appendices are published online only. To view these files please visit the journal online (http://thorax.bmj. com/content/67/8.toc)

${ }^{1}$ Centre for Infection and Immunity, Queen's University of Belfast, Belfast, UK

${ }^{2}$ Institute for Lung Health, Department of Infection, Inflammation and Immunity, University of Leicester,

Leicester, UK

${ }^{3}$ Royal Brompton and Harefield NHS Foundation Trust, London, UK

${ }^{4}$ The University of Manchester (MAHSC) and University Hospital of South Manchester. UK

${ }^{5}$ Centre for Public Health, Queen's University of Belfast, Belfast, UK

\section{Correspondence to}

Professor Liam G Heaney, Centre for Infection and Immunity, Queen's University of Belfast, Level 8, Belfast City Hospital, Lisburn Road, Belfast BT9 7AB, UK

I.heaney@qub.ac.uk

Received 6 March 2012 Accepted 30 March 2012 Published Online First

11 May 2012

\section{(2) UNLOCKA}

This paper is freely available online under the BMJ Journals unlocked scheme, see http:// thorax.bmj.com/site/about/ unlocked.xhtml

AUDIT UPDATE

\title{
Clinical management and outcome of refractory asthma in the UK from the British Thoracic Society Difficult Asthma Registry
}

\author{
Joan Sweeney, ${ }^{1}$ Chris E Brightling, ${ }^{2}$ Andrew Menzies-Gow, ${ }^{3}$ Robert Niven, ${ }^{4}$ \\ Chris C Patterson, ${ }^{5}$ Liam G Heaney, ${ }^{1}$ on behalf of the British Thoracic Society Difficult \\ Asthma Network
}

\section{ABSTRACT}

Refractory asthma represents a significant unmet clinical need. Data from a national online registry audited clinical outcome in 349 adults with refractory asthma from four UK specialist centres in the British Thoracic Society Difficult Asthma Network. At follow-up, lung function improved, with a reduction in important healthcare outcomes, specifically hospital admission, unscheduled healthcare visits and rescue courses of oral steroids. The most frequent therapeutic intervention was maintenance oral corticosteroids and most steroid sparing agents (apart from omalizumab) demonstrated minimal steroid sparing benefit. A significant unmet clinical need remains in this group, specifically a requirement for therapies which reduce systemic steroid exposure.

\section{BACKGROUND}

We have previously published the clinical features of a well characterised group of patients with refractory asthma from specialist UK centres operating established dedicated multidisciplinary assessment protocols and identified important differences between patient groups in individual centres. ${ }^{1}$ Using the national online registry, we have now audited clinical outcome in 349 of the 382 patients in the original cohort (median followup 3.1 years, IOR 1.9-5.5).

\section{RESULTS}

There were no differences in baseline demographical variables in the follow-up cohort compared with those lost to follow-up (online supplementary appendix 1).

At follow-up, there was a significant improvement in lung function and a reduction in important healthcare outcomes, specifically hospital admission, unscheduled healthcare visits and rescue courses of oral steroids (table 1). These effects were generally consistent across clinical centres though unscheduled visits were significantly increased in Manchester (online supplementary appendix 2), reflecting the delivery of care at this centre where patients with an increase in symptoms are encouraged to attend the hospital centre

There was no significant change in dose of inhaled steroid or reported reliever medication use but there was a significant increase in the number of patients prescribed maintenance oral steroids (from $146(42 \%)$ to $199(57 \%)$ ). Only 25 patients (7\%) successfully withdrew oral steroids, whereas $78(22 \%)$ were moved onto maintenance oral steroids. There was no significant difference in the dose of oral steroids from baseline to follow-up (16.2 $\pm 10.4 \mathrm{mg}$ baseline, 15.3 \pm 12.8 follow-up)

Consistent with this widespread use of oral steroids, there was a significant reduction in blood eosinophils and increase in body mass index (BMI) (table 1). There was a non-significant trend for the subjects on oral steroids at follow-up to have a higher BMI compared with those not on oral steroids (subjects not on oral steroids 29.5 \pm 7.0 , subjects on oral steroids 30.9 $\pm 6.0, p=0.07$ ). However, BMI also increased in patients not on maintenance steroids at follow-up (baseline BMI $28.3 \pm 6.8$ vs follow-up BMI 29.2 $\pm 6.9, p<0.001$ ); in this group median rescue steroid exposure was one course of steroids in the preceding 12 months (IOR $0-3)$.

While blood eosinophils decreased, exhaled nitric oxide paradoxically increased. Because paired fractional exhaled nitric oxide (FeNO) data were only available in a limited number of patients, we examined paired blood eosinophil counts in this subgroup $(n=75)$. The paradoxical fall in blood eosinophils and rise in FeNO were also apparent in this group (eosinophil count in subjects with paired FeNO measurements - baseline eosinophils $\times 10^{9} /$ litre, median 0.33 (IOR 0.12-0.54) vs follow-up eosinophils, median 0.24 (IOR 0.1-0.4), $\mathrm{p}=0.001$; and baseline FeNO ppb, 47 (IOR 22-69) vs followup FeNO, 88 (IOR 76-99), p<0.001).

Steroid sparing strategies (online supplementary appendix 3) and additional therapeutic strategies (online supplementary appendix 4) utilised in this refractory population are shown by centre; therapeutic success was defined by the treating clinician. In general, small numbers of patients were tried on steroid-sparing strategies and few were recorded as clinically beneficial. The use of other interventions was infrequent and variable across clinical centres.

Responders to omalizumab (37 of 59 (63\%) based on criteria for the National Health Service Outcomes Drug Reimbursement Scheme, http:// guidance.nice.org.uk/TA133/Guidance/doc/English) were more likely to be off oral steroids at clinical follow-up (17 of 37 vs 4 of $22, p=0.031$, OR 3.8 
Table 1 Lung function and healthcare outcomes for cohort

\begin{tabular}{|c|c|c|c|}
\hline & Baseline & Follow-up & p Value \\
\hline $\begin{array}{l}\text { Pre-bronchodilator } \mathrm{FEV}_{1} \% \\
\text { predicted (259) }\end{array}$ & $66.4 \pm 23.7$ & $72.7 \pm 26.8$ & $<0.001$ \\
\hline $\begin{array}{l}\text { Pre-bronchodilator FVC \% } \\
\text { predicted (242) }\end{array}$ & $82.7 \pm 20.3$ & $86.5 \pm 21.5$ & 0.002 \\
\hline $\begin{array}{l}\text { Post-bronchodilator } \mathrm{FEV}_{1} \% \\
\text { predicted (77) }\end{array}$ & $79.2 \pm 21.5$ & $77.6 \pm 30.7$ & 0.61 \\
\hline $\begin{array}{l}\text { Post-bronchodilator FVC \% } \\
\text { predicted (72) }\end{array}$ & $90.6 \pm 19.8$ & $86.3 \pm 25.9$ & 0.08 \\
\hline $\begin{array}{l}\text { Rescue oral steroids in } \\
\text { previous } 12 \text { months (302) }\end{array}$ & $4(2-6)$ & $2(0-4)$ & $<0.001$ \\
\hline $\begin{array}{l}\text { Hospital admissions in } \\
\text { previous } 12 \text { months (324) }\end{array}$ & $0(0-2)$ & $0(0-1)$ & $<0.01$ \\
\hline $\begin{array}{l}\text { Unscheduled visits in } \\
\text { previous } 12 \text { months (315) }\end{array}$ & $4(2-6)$ & $2(0-6)$ & $<0.05$ \\
\hline $\begin{array}{l}\text { Inhaled steroid dose, BDP } \\
\text { equivalent (327) }\end{array}$ & $2000(1000-2000)$ & $2000(1200-2000)$ & 0.80 \\
\hline Average daily SABA use (205) & $6(4-9)$ & $8(4-10)$ & 0.058 \\
\hline Blood eosinophils (206) & $0.33(0.11-0.60)$ & $0.20(0.09-0.43)$ & $<0.001$ \\
\hline FeNO (112) & $40(18-69)$ & $89(77-102)$ & $<0.001$ \\
\hline Body mass index & $29.2 \pm 6.5$ & $30.2 \pm 6.4$ & $<0.001$ \\
\hline
\end{tabular}

Group data (mean $\pm S D$ or median (IQR)) for all subjects are presented in column 1 followed by data for individual centres. Comparisons were made using paired samples $t$ tests or Wilcoxon signed rank tests; significance was taken as $p<0.05$.

$\mathrm{BDP}$, beclometasone dipropionate; FeNO, fractional expiratory nitric oxide; $\mathrm{FEV}_{1}$, forced expiratory volume in $1 \mathrm{~s}$; FVC, forced vital capacity; SABA, short-acting $\beta$ agonist.

(95\% CI 1.0 to 18.2$\left.), \chi^{2}\right)$. Individual responses also support a steroid-sparing effect-in subjects on maintenance oral steroids pre omalizumab $(\mathrm{n}=28), 9$ withdrew oral steroids completely (baseline dose $20 \mathrm{mg}(10-35 \mathrm{mg})$ ), 11 had a steroid dose reduction (baseline dose $20 \mathrm{mg}$ (15-20 mg), follow-up $13 \mathrm{mg}$ (10-15 mg), $p=0.003$, Wilcoxon signed rank test) and 8 had an increase in dose (10 $\mathrm{mg}(7-10 \mathrm{mg})$, follow-up 12.25 (10-15 mg), $p=0.027$, Wilcoxon signed rank test). Of the other 8 subjects whose condition responded to omalizumab only 1 progressed to oral steroids, whereas of those on omalizumab whose condition did not respond, 18 of 22 were on oral steroids at follow-up (15 mg (10-25 mg)).

Using logistic regression, baseline predictors of maintenance oral steroids at follow-up were maintenance oral steroids at baseline (OR 8.3, 95\% CI 4.8 to 14.4), male gender (OR 2.2, 95\% CI 1.3 to 3.7 ), and rescue steroids in the preceding year (OR 2.3, $95 \%$ CI 1.1 to 4.9 ). At follow-up, 84 of 127 men (66\%) compared with 115 of 222 women (52\%) were on maintenance oral steroids $\left(\chi^{2}, \mathrm{p}<0.01\right)$

\section{DISCUSSION}

This audit provides the first outcome data on a well characterised cohort of adults with severe refractory asthma. We report significant changes in important healthcare outcomes, particularly reductions in unscheduled visits, hospital admissions and rescue oral steroids, which taken collectively suggests a reduction in severe exacerbations. Improvement was also seen in lung function, but notably daily reliever medication use was not different, suggesting persistent symptomatic morbidity in this group. Because of the precise characterisation of this group, we believe this is due to asthma and not other non-asthma comorbidities.

As this is an observational clinical registry, we cannot exclude the possibility that the improvement in healthcare outcomes simply represents 'regression to the mean', since at the time of referral and initial assessment, patients are likely to be clinically unwell. However, $60-75 \%$ of patients in these centres are tertiary referrals and had difficult asthma for prolonged periods prior to assessment, making regression to the mean unlikely to be the entire explanation for the observed improvement. Even at baseline, in this severe asthmatic population, hospital admission rate was relatively low, but unscheduled healthcare contact and rescue steroid courses were high. With appropriate specialist management, hospital admission rates were further reduced, suggesting the economic cost of refractory asthma is unlikely to be driven by hospital admission.

The commonest therapeutic strategy was initiation of maintenance steroids, which is consistent with the reduction in peripheral blood eosinophils and the reduction in rescue oral steroid courses. The dissociation between FeNO, clinical outcome and blood eosinophilia is consistent with other data, which have shown that FeNO-based strategies have not been able to reduce exacerbation rates. ${ }^{2}$ In a recent oral steroid tapering study, which included FeNO as part of the steroid reduction algorithm, weekly Asthma Control Questionnaire and forced expiratory volume in $1 \mathrm{~s}$ measurement were the major drivers of steroid reduction, with minimal contribution from daily FeNO. ${ }^{3}$ Collectively, these data question whether FeNO is useful in adjusting steroid dose in patients on maintenance or frequent bursts of oral steroids.

The increase in BMI is also consistent with more steroid exposure, but BMI also increased in subjects not on maintenance oral steroids at follow-up. This latter group remained on highdose inhaled steroids but rescue steroid exposure in the preceding 12 months was relatively low (median 1, IOR 0-3), suggesting that BMI increase is not exclusively related to oral steroid exposure in this population, and reduced exercise capacity due to persistent asthma may be relevant.

The best predictor of being on oral steroids was being on them at referral, which might initially suggest that specialist services have a minimal effect on maintenance steroid exposure in this patient population. However, this cohort of patients had well phenotyped refractory asthma after detailed systematic evaluation and issues such as incorrect diagnosis, comorbidities and non-adherence have been identified and these subjects excluded. One of the major advantages and benefits of a specialist difficult asthma service is ensuring precise patient characterisation and appropriateness of high-dose asthma therapy in subjects with refractory asthma. ${ }^{4}$

It is unclear why a greater proportion of men were more likely to be on oral steroids at follow-up. Cohorts of difficult and refractory asthma typically include more women, ${ }^{4}{ }^{5}$ but these data suggest that the requirement for oral steroids, which might be interpreted as one index of severity, is less common in women. Frequency of rescue steroids is also predictive of progression as this identifies someone with steroid-responsive disease prone to exacerbation despite high-dose inhaled therapy. Steroid-sparing strategies (cyclosporin, methotrexate, azathioprine, mycophenolate) are used variably across centres, with limited clinical success. The low trial rate in some centres reflects the potential side effects of these agents, but additionally our observational data also suggest that the success rate of the most commonly used agents (methotrexate and cyclosporin) is low, which is consistent with the conclusions of recent Cochrane reviews of both methotrexate and cyclosporin. 67

The only additional therapeutic intervention that demonstrated a steroid-sparing effect was omalizumab. In subjects who received a clinical trial of omalizumab, the overall response rate was $63 \%$ and $20(71 \%)$ of the 28 on oral steroids either withdrew or significantly reduced their oral steroid dose.

In summary, this audit demonstrates improved outcomes with reduced exacerbation rates and healthcare utilisation, but at the cost of increased numbers of subjects on systemic steroids. 
Steroid-sparing therapies are infrequently used and are only modestly successful in routine clinical practice. In patients who respond to omalizumab, there is the suggestion of a significant steroid-sparing effect in some but not all subjects. There remains a significant unmet clinical need in this group and specifically a requirement for therapies which reduce systemic steroid exposure.

Acknowledgements Thanks to the data input staff and medical and nursing staff in the UK Difficult Asthma Centres-Belfast, Jacqui Gamble and Kirsty Graham; Leicester, Sarah Terry, Amisha Singapuri, Beverley Hargadon, Maria Shelley, Ruth Green, lan Pavord, Andrew Wardlaw, Peter Bradding; London, Markus Hoffman and Suzanne Regan; Manchester, Gill McCumesky and Leanne Holmes.

Contributors LGH is the coordinator of the British Thoracic Society Difficult Asthma Registry and with JS collated and managed the data for this manuscript. CEB and AM-G and RN co-lead the British Thoracic Society Difficult Asthma Network and all have contributed equally to this manuscript.

Funding Pilot funding for the Registry was provided as unrestricted research grants from Astra Zeneca, GlaxoSmithKline, Novartis and Medimmune. Sweeney is supported by HSC R\&D (NI) and GlaxoSmithKline (PhD studentship). CB is supported by a Wellcome Senior Clinical Fellowship.

Competing interests Ms Sweeney is supported by HSC R\&D (NI) and GlaxoSmithKline (PhD studentship funding). Professor Brightling is supported by a Wellcome Senior Clinical Fellowship and has received consultancy fees and or research funding from GlaxoSmithKline, AZ, Medlmmune, Amgen, Novartis, Chiesi, BI and Roche. Dr Menzies-Gow has attended advisory boards for Novartis and Genentech. He has received sponsorship to attend international meetings from GlaxoSmithKline, Novartis and Boeringer Ingelheim. He has received lecture fees from Novartis, GlaxoSmithKline, Astra Zeneca and Chiesi. Dr Niven has received an unrestricted grant of $£ 10000$ from Novartis in 2010 towards development of clinical services at the University Hospital of South Manchester. In addition he has lectured in the field of severe allergic asthma at Novartis-sponsored meetings receiving honoraria in total not exceeding $£ 5000$ in the last 3 years. Dr Niven has also performed lecturing at pharmaceutically sponsored meetings for the following pharmaceutical companies in the last 3 years: Astra Zeneca $(<£ 1000)$, GlaxoSmithKline $(<£ 5000)$ and Chiesi $(<£ 1000)$. He has sat on advisory boards for the following companies in the last
3 years: Vectura, Novartis, GlaxoSmithKline, receiving reimbursement not exceeding $£ 1000$. He has received sponsorship support to attend international academic meetings. Dr Niven (or any members of his family) has no shares or any percuniary interest in any pharmaceutical industry and has nothing to gain financially from the publication of this paper. Dr C Patterson's spouse holds shares in GlaxoSmithKline. Professor Heaney has received grant funding from Genentech, and GlaxoSmithKline, has taken part in Advisory Boards and given lectures at meetings supported by GlaxoSmithKline, Merck Sharpe \& Dohme, Nycomed, Novartis and Astra Zeneca. He has received support funding to attend International Respiratory meetings (Astra Zeneca, Chiesi, Novartis, Teva and GlaxoSmithKline) and has taken part in asthma clinical trials (GSK and Genentech) for which his Institution was remunerated. None of these activities have any direct relationship to the content of this manuscript.

Ethics approval Ethics approval was provided by ORECNI.

Provenance and peer review Not commissioned; externally peer reviewed.

\section{REFERENCES}

1. Heaney LG, Brightling CE, Menzies-Gow A, et al. Refractory asthma in the UK: cross sectional findings from a UK multicentre registry. Thorax 2010;65: 787-94.

2. Petsky HL, Cates CJ, Lasserson TJ, et al. A systematic review and meta-anslysis: tailoring asthma treatment on eosinophilic markers (exhaled nitric oxide or sputum eosinophils). Thorax 2012;67:199-208.

3. Hashimoto S, Brinke AT, Roldaan AC, et al. Internet-based tapering of oral corticosteroids in severe asthma: a pragmatic randomised controlled trial. Thorax 2011;66:514-20.

4. Heaney LG, Conway E, Kelly C, et al. Predictors of therapy resistant asthma: outcome of a systematic evaluation protocol. Thorax 2003:58:561-6.

5. Moore WC, Bleecker ER, Curran-Everett D, et al; For the National Heart, Lung, and Blood Institute's Severe Asthma Research Program. Characterization of the severe asthma phenotype by the National Heart, Lung, and Blood Institute's Severe Asthma Research Program. J Allergy Clin Immunol 2007;119:405-13.

6. Evans DJ, Cullinan P, Geddes DM, et al. Cyclosporin as an oral corticosteroid sparing agent in stable asthma. Cochrane Airways Group 2001;2:CD002993. doi:10.1002/ 14651858.CD002993

7. Davies HRH R, Olson LLG, Gibson PG. Methotrexate as a steroid sparing agent for asthma in adults. Cochrane Airways Group. Published Online First: 21 January 2009 doi:10.1002/14651858.CD000391 